\title{
Assessment of Liver Fibrosis in Non-Alcoholic Fatty Liver Disease Using Acoustic Radiation Force Impulse
}

\author{
Nader S. Anis ${ }^{\text {a }}$, Ahmed F. Yousef ${ }^{b}$, Hamada M. Khater ${ }^{\text {b }}$, Enas M. El-Kharbotly ${ }^{\mathrm{c}}$ \\ a Department of Radiology, \\ Damanhour Oncology Center, \\ Egypt, b Department of \\ Radiology, Benha faculty of \\ medicine, Benha University, \\ Egypt. c Department of \\ hepatology, Gastroenterology \\ and Infectious Diseases Faculty \\ of Medicine, Benha University, \\ Egypt. \\ Correspondence to: Nader S. \\ Anis, Department of Radiology, \\ Damanhour Oncology Center, \\ Egypt. \\ Email: \\ nido10@msn.com \\ Received: 31 August 2020 \\ Accepted: 22 September 2020

\begin{abstract}
:
Background: The clinical importance of NAFLD and the limitations of liver biopsy have increased the need for accurate and noninvasive imaging methods to evaluate NAFLD. Acoustic radiation force impulse (ARFI) imaging is a new and promising ultrasound-based diagnostic technique that, evaluating the wave propagation speed, allows the assessment of the tissue stiffness. Aim of study: Assess the reliability of acoustic radiation force impulse (ARFI) for diagnosis of liver fibrosis in patients with nonalcoholic fatty liver disease (NAFLD) compared with NAFLDfibrosis score and FIB-4 Index. Methods: This study was conducted on 100 patients diagnosed with NAFLD and 30 healthy subjects referred from Damanhour Medical National Institute outpatient clinics, from June 2018 to December 2018. Results: The degree of agreement between ARFI and FIB-4 index is $80 \%$. The ROC curve for the performance of ARFI in prediction of F3 or more (taking FIB-4 as a gold standard) shows sensitivity of $100 \%$ and specificity of $79.5 \%$. It also showed that the degree of
\end{abstract} \\ agreement between ARFI and NAFLD fibrosis score is $87.1 \%$. The ROC curve for the \\ performance of ARFI in prediction of F3 or more (taking NAFLD Fibrosis Score as a gold \\ standard) shows sensitivity of $71.4 \%$ and specificity of $83.3 \%$. Conclusion: The degree of \\ agreement between ARFI on one hand and FIB-4 score and NAFLD fibrosis score on the other \\ hand is acceptably high and is statistically significant.
}

Key words: NAFLD, ARFI, FIB-4, Elastography. 



\section{Introduction}

Nonalcoholic fatty liver disease (NAFLD) is the name given to a spectrum of liver disorders associated with hepatic steatosis that is not due to significant alcohol consumption or other secondary causes, such as steatogenic medication, or inborn errors of metabolism. This disorder encompasses a wide range of diseases, from simple steatosis, which is relatively benign, to hepatic inflammation, hepatocyte injury, and fibrosis, a syndrome referred to as nonalcoholic steatohepatitis (NASH), which can progress to cirrhosis. Over the last three decades, NAFLD has emerged as one of the leading causes of cirrhosis, and a large proportion of individuals who previously had been classified as having cryptogenic cirrhosis are now believed to have cirrhosis due to NASH. (1)

Liver biopsy is regarded as the gold standard for the assessment of NAFLD and is the only reliable method for differentiating NASH from simple steatosis. This method, however, is invasive and is, therefore, unsuitable for screening large numbers of subjects at risk, or for follow-up of patients with NAFLD after therapeutic intervention. Furthermore, as liver biopsy samples are small in size, they are subject to sampling variability.(2)
Ultrasound elastography (USE) is an imaging technology sensitive to tissue stiffness that was first described in the 1990s. (3). It has been further developed and refined in recent years to enable quantitative assessments of tissue stiffness. Elastography methods take advantage of the changed elasticity of soft tissues resulting from specific pathological or physiological processes. (4)

\section{Patients and methods:}

This study was conducted on 100 patients diagnosed with NAFLD and 30 healthy subjects referred from Damanhour Medical National Institute outpatient clinics. The diagnosis of NAFLD was established according to the practice guidelines of the American Association for the Study of Liver Diseases ( AASLD ) (5) as evidence of hepatic steatosis on imaging in the absence of other causes of liver disease.

Healthy controls included individuals with no clinical, imaging or laboratory evidence of hepatic steatosis or any other chronic liver disease.

All patients were subjected to complete history taking, clinical examination, laboratory investigation (NAFLD fibrosis score and FIB-4 Index were calculated) and 
radiological investigation (Abdominal ultrasound and AFRI).

The NAFLD fibrosis score was calculated using available variables (age, BMI, hyperglycemia, platelet count, albumin, and AST/ALT ratio) and is calculated using this formula:

$$
\begin{aligned}
& (-1.675+0.037 \times \text { age }(\text { years }))+(0.094 \times \text { BMI } \\
& (\mathrm{kg} / \mathrm{m} 2))+(1.13 \times \mathrm{IFG} / \text { diabetes }(\text { yes }=1, \text { no }=0)) \\
& +(0.99 \times \text { AST/ALT ratio })-(0.013 \times \text { platelet } \\
& \left.\left(\times 10^{9} / \mathrm{L}\right)\right)-(0.66 \times \text { albumin }(\mathrm{g} / \mathrm{dL})) .(6)
\end{aligned}
$$

NAFLD score $\leq \mathbf{- 1 . 4 5 5}=$ less probability of fibrosis.

NAFLD score -1.455-0.675 = indeterminate score.

NAFLD score $>\mathbf{0 . 6 7 5}=$ high probability of fibrosis.

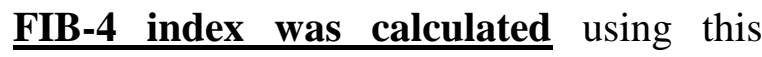
formula:

Age (years) $\times$ AST (U/L)/[PLT $\left(10^{9} / \mathrm{L}\right) \times \mathrm{ALT}^{1 / 2}(\mathrm{U} / \mathrm{L}) .(7)$

FIB-4 score $<1.45=$ Less probability of fibrosis.

FIB-4 score $1.45-3.25=$ indeterminate score.

FIB-4 $>3.25=$ High probability of fibrosis.

Abdominal ultrasound using Acuson s3000; Siemens is used for liver scanning. A curvilinear array transducer, 5-2 $\mathrm{MHz}$ was used.

Patients were required to fast for at least six hours prior to the examination, abstain from smoking or chewing gum in the same period.
ARFI was done with the patient in the supine or slight $\left(30^{\circ}\right)$ left lateral decubitus position. The right arm is raised overhead to increase the intercostal acoustic window. The probe was placed in an intercostal position. The B-mode image was optimized for the "best acoustic window" to provide the best results. The ARFI pulse was made perpendicular to the liver capsule to limit refraction of the pulse. The measurement was performed while the patient holds his or her breath. Taking a deep breath or using a Valsalva maneuver or deep expiration changes hepatic venous pressures that can affect the stiffness measurements. (8)

The literature suggests that 10 measurements should be obtained and the median reported. More than $60 \%$ of the measurements should be "good" measurements; if not, a value should not be reported. A "good" measurement is one where a numerical result is obtained, not an "x.xx" or "0.00." In some studies, investigators suggest that a smaller number of measurements may have similar accuracy. In this study, six measurement for each patient were taken. (9) 


\section{$\underline{\text { ARFI values }}$}

Optimal cut-offs of ARFI for the diagnosis of liver fibrosis.(10)

\begin{tabular}{|l|l|}
\hline F1 $\geq$ & 1.185 \\
\hline F2 $\geq$ & 1.215 \\
\hline F3 $\geq$ & 1.54 \\
\hline F4 $\geq$ & 1.94 \\
\hline
\end{tabular}

In this study, F1 and F2 were considered

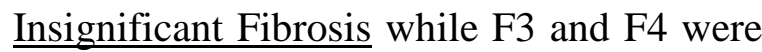
considered Significant Fibrosis.

Exclusion criteria: Age less than 18 years, patients with significant alcohol consumption, patients with Hepatitis C positive, patients with Hepatitis B positive, patients with symptoms and signs suggesting: Haemochromatosis, Wilson's disease, Alpha-one anti-trypsin deficiency. Patients with Autoimmune hepatitis, patient with history intake of steatogenic medications, patients with decompensated cirrhosis, patients with heart failure, patients with renal failure and Pregnancy.

Duration of the study: This study was done in a period of six months from June 2018 to December 2018.

Statistical analysis: The collected data were tabulated and analyzed using SPSS version 16 software (SpssInc, Chicago, ILL
Company. Categorical data were presented as number and percentages while quantitative data were expressed as mean \pm standard deviation median, IQR and range. Chi square test $\left(\mathrm{X}^{2}\right)$, or Fisher's exact test (FET) were used to analyze categorical variables. Coordinate of correlation was assessed by Cohen Kappa test to assess degree of agreement between 2 raters. Quantitative data were tested for normality using Shapiro-Wilks test, assuming normality at $\mathrm{P}>0.05$. Student " $t$ " test was used to analyze normally distributed variables among 2 independent groups. While non parametric variables were analyzed using Man Whitney U test. Difference among 3 independent means was analyzed using Kruskal Wallis test (KW) for non-parametric variables. Significant KW tests was followed by post hoc multiple comparisons using Bonferroni test to detect the significant pairs. Spearman's correlation coefficient (rho) was used to assess correlations. ROC curve analysis was constructed to assess the performance of ARFI in prediction of fibrosis among patient's group. The accepted level of significance in this work was stated at 0.05 ( $\mathrm{P}<0.05$ was considered significant). 


\section{Results}

Patient Demographics: regarding the age, the mean age of patient group was $46.8 \pm 11.2$ while in the control was $43.1 \pm 9.9$ with no significant difference between both groups $(\mathrm{P}$ value $=0.102(\mathrm{NS})$. Regarding the sex, $54 \%$ of the patient's group were males while $50 \%$ of the control were females with no significant difference between both groups $(\mathrm{P}$ value $=0.70(\mathrm{NS}))$. Regarding diabetes, $28 \%$ of the patient's group were diabetic while no one in the control group were diabetic with high significant difference between both groups ( $\mathrm{P}$ value $=0.001(\mathrm{HS}))$. Table 1 .

Anthropometric measurement of the studied groups: There was high significant difference between patient and control group as regard weight and $\mathrm{BMI}(\mathrm{P}$ value $=$ $<0.001)$. There was no significant difference between patient and control groups as regard height $(\mathrm{P}$ value $=0.27)$. Table 2, fig. 1

FIB-4 score of the studied group: $73 \%$ of the cases group and $96.7 \%$ of the control group had insignificant fibrosis (F0-F2). 2\% of the cases group and no one in the control group had significant fibrosis (F3-F4). $25 \%$ of the cases group and $3.3 \%$ of the control group were indeterminate. There was significant difference between cases group and controls group $(\mathrm{P}$ value $=\mathbf{0 . 0 1 3})$. Table 3.

NAFLD Fibrosis score of the studied group: $48 \%$ of the cases group and $96.7 \%$ of the control group had insignificant fibrosis (F0-F2). 14\% of the cases group and no one in the control group had significant fibrosis (F3-F4). 38\% of the cases group and $3.3 \%$ of the control group were indeterminate. There was high significant difference between cases group and controls group (P value $<0.001$ ). Table 4.

ARFI Grade of the studied group: $54 \%$ of the cases and $53.3 \%$ of the control showed F0 score. $4 \%$ of the cases and $40 \%$ of the control showed F1 score. 10\% of the cases and $3.3 \%$ of the control showed F2 score. $14 \%$ of the cases and $3.3 \%$ of the control showed F3 score. 18\% of the cases and no one of the control showed F4 score. There was high significant difference between both groups ( $\mathrm{P}$ value $<0.005)$. table 5 .

\section{Degree of agreement between ARFI and FIB-4 index (while omitting indetermined): there was $80 \%$ agreement between both scores. Table 6 . \\ Degree of agreement between ARFI and NAFLD Fibrosis score (while omitting indetermined): there was $87.1 \%$ agreement between both scores. Table 7 .}


Degree of agreement between Fib-4 and

NAFLD results: there was $63 \%$ agreement between both scores. Table 8 .

\section{Relation between grade of fatty liver and}

ARFI: $30 \%$ of grade I fatty liver, $26.7 \%$ of grade II fatty liver and $37.1 \%$ of grade III fatty liver patients showed significant fibrosis (>/=F3) according to ARFI. There was no significant difference between them $(\mathrm{P}$ value $=0.76(\mathrm{NS})$. Table 9 .

Table (1): a comparison between patients and controls as regard age, sex and diabetes.

\begin{tabular}{|c|c|c|c|c|c|c|c|}
\hline \multicolumn{2}{|c|}{ Variable } & \multicolumn{2}{|c|}{ Patients $(n=100)$} & \multicolumn{2}{|c|}{ Controls $(n=30)$} & St. "t" te & $\mathbf{P}$ \\
\hline \multirow[t]{3}{*}{ Age (ys) } & Mean \pm SD & \multicolumn{2}{|c|}{$46.8 \pm 11.2$} & \multicolumn{2}{|c|}{$43.1 \pm 9.9$} & 1.64 & 0.102 (NS) \\
\hline & Range & \multicolumn{2}{|c|}{$25-74$} & \multicolumn{2}{|c|}{$21-61$} & & \\
\hline & & No. & $\%$ & No. & $\%$ & $\mathbf{X}^{2}$ & $\mathbf{P}$ \\
\hline \multirow[t]{2}{*}{ Sex } & Male & 54 & 54.0 & 15 & 50.0 & \multirow[t]{2}{*}{0.15} & \multirow[t]{2}{*}{0.70 (NS) } \\
\hline & Female & 46 & 46.0 & 15 & 50.0 & & \\
\hline \multirow{2}{*}{ DM } & Non diabetic & 72 & 72.0 & 30 & 100.0 & \multirow{2}{*}{10.7} & \multirow{2}{*}{$=0.001(\mathrm{HS})$} \\
\hline & Diabetic & 28 & 28.0 & 0 & 0.0 & & \\
\hline
\end{tabular}

Table (2) shows a comparison between patients and controls as regard weight, height and BMI.

\begin{tabular}{|c|c|c|c|c|c|c|c|c|}
\hline \multirow[t]{2}{*}{ Variable } & \multicolumn{3}{|c|}{ Patients $(n=100)$} & \multicolumn{3}{|c|}{ Controls $(n=30)$} & \multirow[t]{2}{*}{ St. "t" test } & \multirow[t]{2}{*}{$\mathbf{P}$} \\
\hline & Mean & \pm SD & Range & Mean & \pm SD & Range & & \\
\hline Weight (kg) & 93.9 & 16.8 & $68-145$ & 75.0 & 13.22 & $46-104$ & 5.61 & $<0.001(\mathrm{HS})$ \\
\hline Height (cm) & 167.6 & 6.2 & $154-185$ & 169.1 & 6.72 & $158-190$ & 1.1 & 0.27 (NS) \\
\hline BMI $\left(\mathrm{kg} / \mathrm{m}^{2}\right)$ & 33.3 & 5.38 & $24.8-49.6$ & 26.1 & 3.62 & $18.4-34.0$ & 6.86 & $<0.001(\mathrm{HS})$ \\
\hline
\end{tabular}




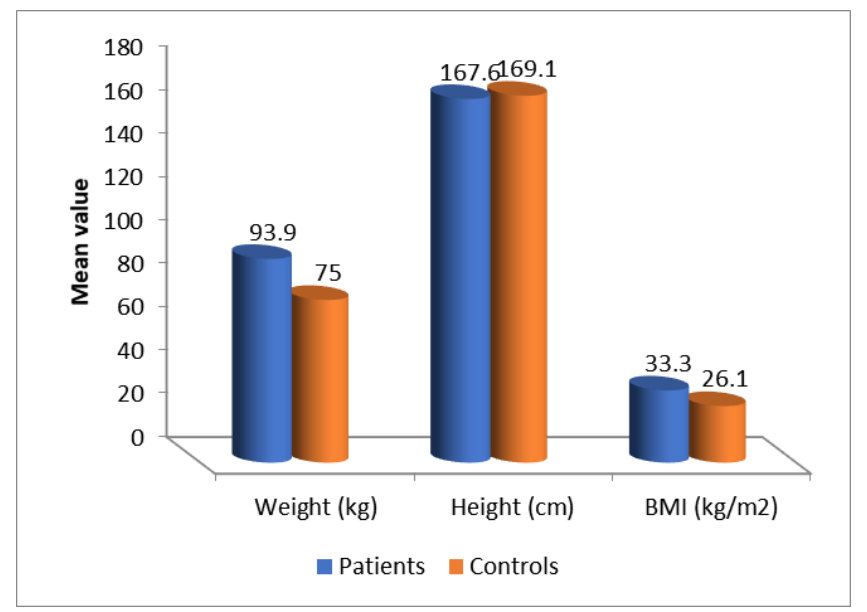

Fig.( 1):Bar chart shows comparison between patients and controls as regard weight, height and BMI.

Table (3): Shows the FIB-4 index of the studied group.

\section{Group}

\section{Cases Controls Total}

\begin{tabular}{|c|c|c|c|c|c|}
\hline & & & Cases & Controls & Total \\
\hline \multirow[t]{6}{*}{ FIB-4 } & F0-F2 & Count & 73 & 29 & 102 \\
\hline & & $\%$ within Group & $73.0 \%$ & $96.7 \%$ & $78.5 \%$ \\
\hline & indeterminant & Count & 25 & 1 & 26 \\
\hline & & $\%$ within Group & $25.0 \%$ & $3.3 \%$ & $20.0 \%$ \\
\hline & F3-F4 & Count & 2 & 0 & 2 \\
\hline & & $\%$ within Group & $2.0 \%$ & $.0 \%$ & $1.5 \%$ \\
\hline \multirow[t]{2}{*}{ Total } & & Count & 100 & 30 & 130 \\
\hline & & $\%$ within Group & $100.0 \%$ & $100.0 \%$ & $100.0 \%$ \\
\hline
\end{tabular}


Table (4) Shows the NAFLD score of the studied group.

\begin{tabular}{|c|c|c|c|c|c|}
\hline & & & \multicolumn{2}{|c|}{ Group } & \multirow[b]{2}{*}{ Total } \\
\hline & & & Cases & Controls & \\
\hline \multirow{6}{*}{$\begin{array}{l}\text { NAFLD Fibrosis } \\
\text { Score }\end{array}$} & F0-F2 & Count & 48 & 29 & 77 \\
\hline & & \% within Group & $48.0 \%$ & $96.7 \%$ & $59.2 \%$ \\
\hline & indeterminant & Count & 38 & 1 & 39 \\
\hline & & \% within Group & $38.0 \%$ & $3.3 \%$ & $30.0 \%$ \\
\hline & F3-F4 & Count & 14 & 0 & 14 \\
\hline & & \% within Group & $14.0 \%$ & $.0 \%$ & $10.8 \%$ \\
\hline \multirow[t]{2}{*}{ Total } & & Count & 100 & 30 & 130 \\
\hline & & \% within Group & $100.0 \%$ & $100.0 \%$ & $100.0 \%$ \\
\hline
\end{tabular}

Table (5) compare the studied groups regarding the ARFI grade

\begin{tabular}{lllccc}
\hline & & & \multicolumn{2}{c}{ Group } & \\
& & & Cases & Controls & Total \\
\hline ARFI grade & F0 & Count & 54 & 16 & 70 \\
& & \% within Group & $54.0 \%$ & $53.3 \%$ & $53.8 \%$ \\
& F1 & Count & 4 & 12 & 16 \\
& & \% within Group & $4.0 \%$ & $40.0 \%$ & $12.3 \%$ \\
& F2 & Count & 10 & 1 & 11 \\
& & \% within Group & $10.0 \%$ & $3.3 \%$ & $8.5 \%$ \\
& Count & 14 & 1 & 15 \\
& \% within Group & $14.0 \%$ & $3.3 \%$ & $11.5 \%$ \\
& Count & 18 & 0 & 18 \\
& & \% within Group & $18.0 \%$ & $0 \%$ & $7.7 \%$ \\
& & Count & 100 & 30 & 130 \\
\hline & \% within Group & $100.0 \%$ & $100.0 \%$ & $100.0 \%$ \\
\hline
\end{tabular}




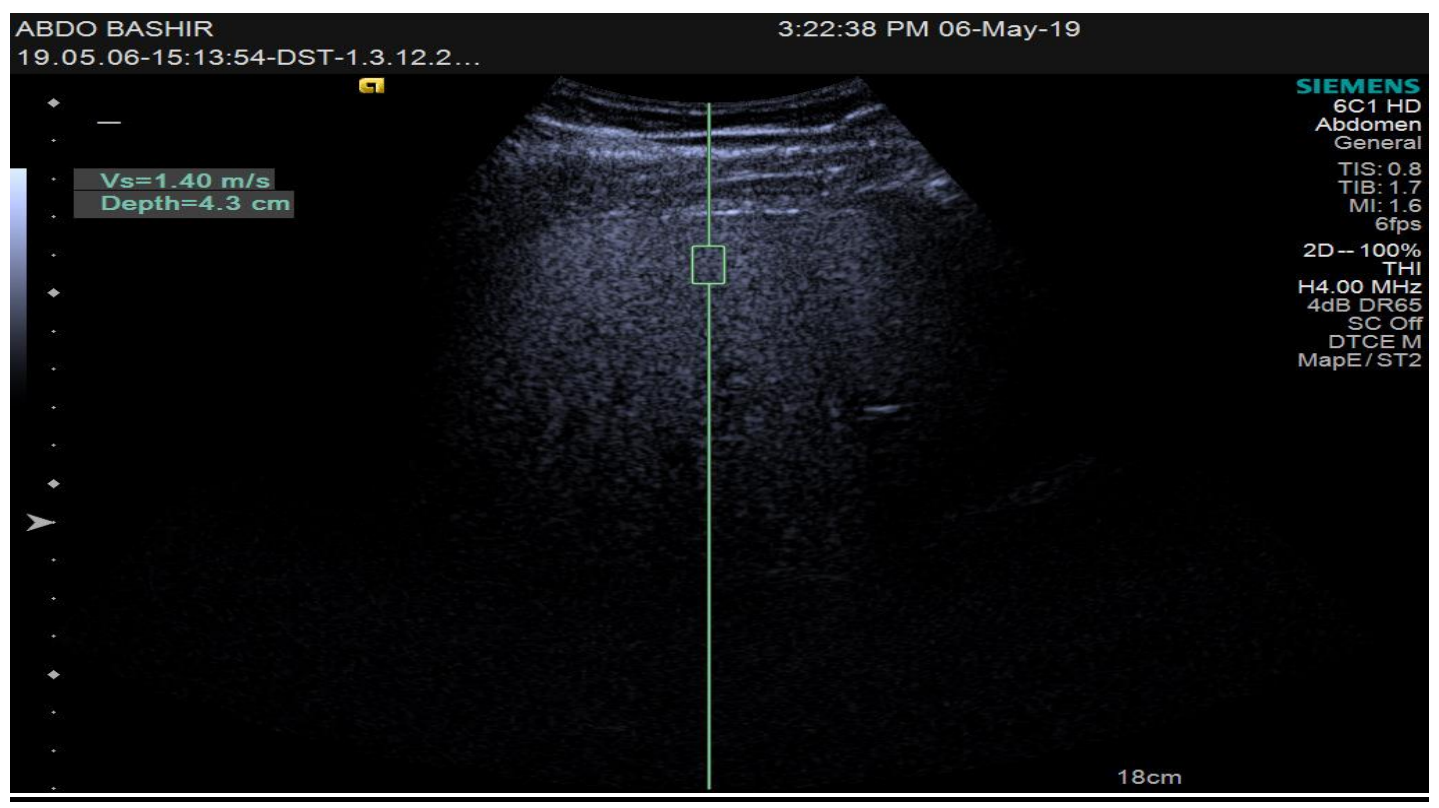

Fig. (2): showing Ultrasound image of ARFI measurement with region of interest (Flexible metering box). A shear wave velocity of $1.40 \mathrm{~m} / \mathrm{s}$ corresponding to $\mathrm{F} 2$ score.

Table (6) shows the degree of agreement between ARFI and FIB-4 index

\begin{tabular}{llllll}
\hline & & & FIB-4 & & \\
& & & $<\mathbf{F 3}$ & $>/=\mathbf{F 3}$ & Total \\
\hline ARFI & $<\mathbf{F 3}$ & Count & 58 & 0 & 58 \\
& & \% within FIBF3 & $79.5 \%$ & $.0 \%$ & $77.3 \%$ \\
& $>/=\mathbf{F 3}$ & Count & $\underline{\mathbf{1 5}}$ & 2 & 17 \\
& & \% within FIBF3 & $20.5 \%$ & $100.0 \%$ & $22.7 \%$ \\
& & Count & 73 & 2 & 75 \\
\hline
\end{tabular}

Kappa test $=0.171 \quad \mathrm{P}=0.008(\mathrm{~S})$


ROC curve for the performance of ARFI in prediction of F3 or more (taking FIB-4 as a gold standard)

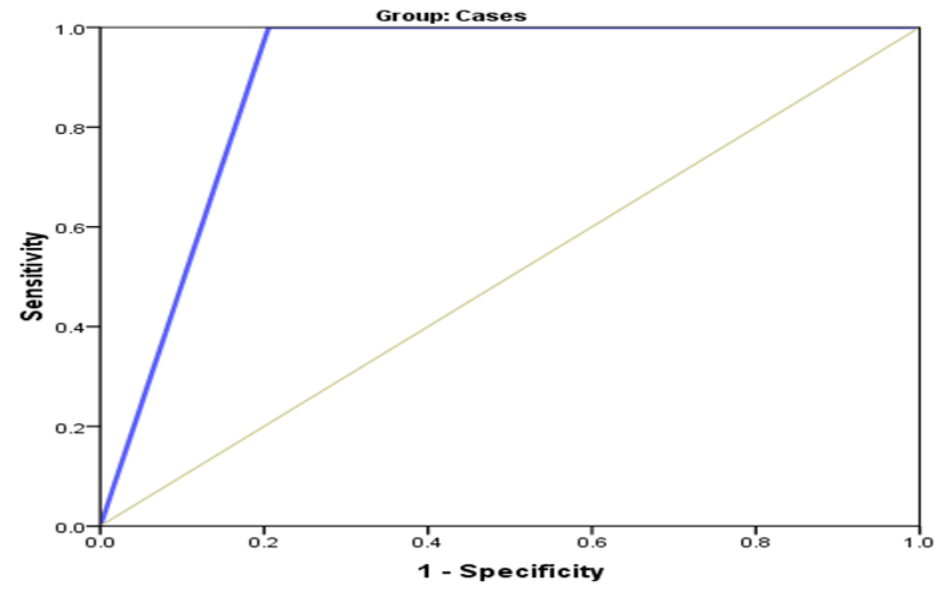

\begin{tabular}{lcccccccc}
\hline Variable & Sens\% & Spec\% & PPV\% & NPV\% & Accuracy\% & AUC & 95\%CI & P \\
ARFI $\geq$ F3 & $100 \%$ & $79.5 \%$ & $11.7 \%$ & $100 \%$ & $80 \%$ & 0.897 & $0.79-1.0$ & $0.049(\mathrm{~S})$ \\
\hline
\end{tabular}

Table (7) shows the degree of agreement between ARFI and NAFLD fibrosis score

\begin{tabular}{lllrrr}
\hline & & \multicolumn{4}{c}{ NAFLD } \\
& & $<\mathbf{F 3}$ & $>/=\mathbf{F 3}$ & \multicolumn{1}{c}{ Total } \\
\hline ARFI & $<\mathbf{F 3}$ & Count & 40 & 4 & 44 \\
& & \% within NAFLDF3 & $83.3 \%$ & $28.6 \%$ & $71.0 \%$ \\
& $>/=\mathbf{F 3}$ & Count & 8 & 10 & 18 \\
& & \% within NAFLDF3 & $16.7 \%$ & $71.4 \%$ & $29.0 \%$ \\
Total & Count & 48 & 14 & 62 \\
& & \% within NAFLDF3 & $100.0 \%$ & $100.0 \%$ & $100.0 \%$ \\
\hline
\end{tabular}


ROC curve for the performance of ARFI in prediction of F3 or more (taking NAFLD as a gold standard)

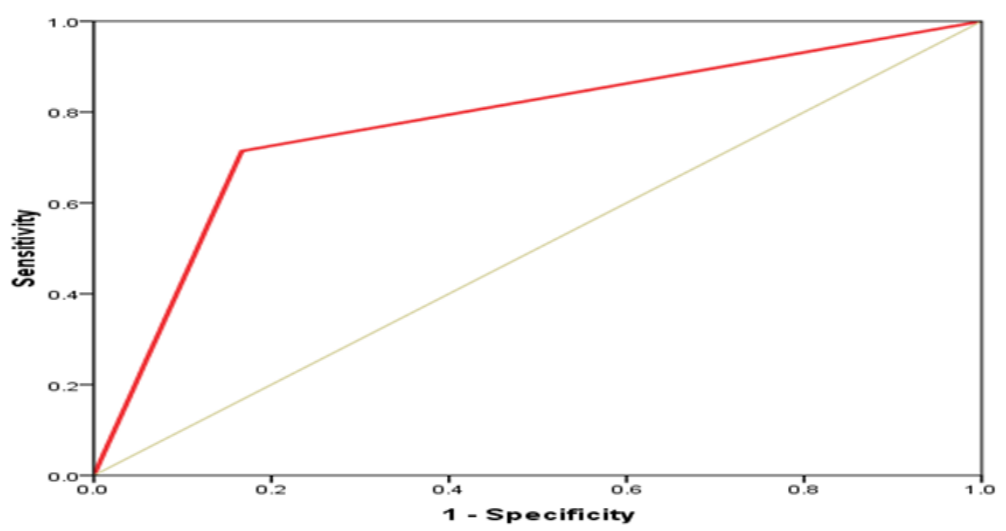

\begin{tabular}{rllllllll}
\hline Variable & Sens $\%$ & Spec\% & PPV \% & NPV \% & Accuracy \% & AUC & 95\%CI & P \\
ARFI $\geq$ F3 & $71.4 \%$ & $83.3 \%$ & $55.6 \%$ & $90.9 \%$ & $87.1 \%$ & 0.774 & $0.62-0.93$ & $0.002(\mathrm{~S})$ \\
\hline
\end{tabular}

Table (8) shows the degree of agreement between FIB-4 and NAFLD fibrosis score

\begin{tabular}{|c|c|c|c|c|c|c|}
\hline & & & \multicolumn{3}{|c|}{ NAFLD Fibrosis Score } & \multirow[b]{2}{*}{ Total } \\
\hline & & & F0-F2 & indeterminant & F3-F4 & \\
\hline \multirow[t]{6}{*}{ FIB-4 score } & F0-F2 & Count & 46 & 23 & 4 & 73 \\
\hline & & $\%$ & $95.8 \%$ & $60.5 \%$ & $28.6 \%$ & $73.0 \%$ \\
\hline & indetermined & Count & 2 & 15 & 8 & 25 \\
\hline & & $\%$ & $4.2 \%$ & $39.5 \%$ & $57.1 \%$ & $25.0 \%$ \\
\hline & F3-F4 & Count & 0 & 0 & 2 & 2 \\
\hline & & $\%$ & $.0 \%$ & $.0 \%$ & $14.3 \%$ & $2.0 \%$ \\
\hline \multirow[t]{2}{*}{ Total } & & Count & 48 & 38 & 14 & 100 \\
\hline & & $\%$ & $100.0 \%$ & $100.0 \%$ & $100.0 \%$ & $100.0 \%$ \\
\hline
\end{tabular}

Kappa test $=0.329$

$\mathrm{P}<0.001(\mathrm{HS})$ 
Table (9) shows relation between grade of fatty liver and ARFI.

\section{US}

Grade 1 fatty liver Grade 2 fatty liver

Grade 3 fatty liver

Total

\begin{tabular}{|c|c|c|c|c|c|c|}
\hline \multirow[t]{4}{*}{ ARFI } & $<$ F3 & Count & 35 & 11 & 22 & 68 \\
\hline & & $\%$ within US & $70.0 \%$ & $73.3 \%$ & $62.9 \%$ & $68.0 \%$ \\
\hline & $>/=\mathbf{F 3}$ & Count & 15 & 4 & 13 & 32 \\
\hline & & \% within US & $30.0 \%$ & $26.7 \%$ & $37.1 \%$ & $32.0 \%$ \\
\hline \multirow[t]{2}{*}{ Total } & & Count & 50 & 15 & 35 & 100 \\
\hline & & $\%$ within US & $100.0 \%$ & $100.0 \%$ & $100.0 \%$ & $100.0 \%$ \\
\hline
\end{tabular}

FET $=0.71 \quad \mathrm{P}=0.76(\mathrm{NS})$

\section{Discussion}

The definition of nonalcoholic fatty liver disease (NAFLD) requires that there is evidence of hepatic steatosis, either by imaging or by histology and there are no causes for secondary hepatic fat accumulation such as significant alcohol consumption, use of steatogenic medication or hereditary disorders. In the majority of patients, NAFLD is associated with metabolic risk factors such as obesity, diabetes mellitus, and dyslipidemia. NAFLD is histologically further categorized into nonalcoholic fatty liver (NAFL) and nonalcoholic steatohepatitis (NASH). NAFL is defined as the presence of hepatic steatosis with no evidence of hepatocellular injury in the form of ballooning of the hepatocytes. NASH is defined as the presence of hepatic steatosis and inflammation with hepatocyte injury (ballooning) with or without fibrosis.(5)

Liver biopsy is regarded as the gold standard for the assessment of NAFLD and is the only reliable method for differentiating NASH from simple steatosis. This method, however, is invasive and is, therefore, unsuitable for screening large numbers of subjects at risk, or for follow-up of patients with NAFLD after therapeutic intervention. Furthermore, as liver biopsy samples are 
small in size, they are subject to sampling variability.(2)

There has been intense interest in noninvasive methods and biomarkers to identify advanced fibrosis in patients with NAFLD such as the NAFLD Fibrosis Score, FIB-4 Index and ARFI. (11)

Acoustic radiation force impulse (ARFI) imaging is a new and promising ultrasoundbased diagnostic technique that, evaluating the wave propagation speed, allows the assessment of the tissue stiffness.(12)

ARFI is implemented in the ultrasound scanner and by using a conventional probe, without any need for external compression so reducing the operator dependency, it evaluates deep tissues stiffness providing complementary information potentially useful for the diagnosis.(13)

The aim of this study is to assess the applicability and reliability of acoustic radiation force impulse (ARFI) for diagnosis of liver fibrosis in patients with nonalcoholic fatty liver disease (NAFLD) compared with NAFLD-fibrosis score and FIB-4 Index.

Patients and controls were matched to each other as regard age and sex (Table 1). This excludes age and sex as 'confounding' factor at any further comparison. In the same table (Table 1), it is clear that diabetes mellitus is significantly more prevalent among patients than controls. This of course reflects the pattern of selection of cases and controls in this study. According to Collantes, Ong, and Younossi 2006, T2DM and insulin resistance have contributed to the development of NAFLD. they have described a higher prevalence of NAFLD among patients with T2DM compared with nondiabetics, with prevalence estimated to be $40 \%$ to $69.5 \% .(14)$

In table (2) and chart (1), obesity as gauged by BMI was significantly more prevalent among patients than controls. This finding is, of course, natural and expected from the pattern of selection of cases and controls. Boza et al. 2005 stated that obesity is a common and well documented risk factor for NAFLD. Both excessive BMI and visceral obesity are recognized risk factors for NAFLD. In patients with severe obesity undergoing bariatric surgery, the prevalence of NAFLD can exceed $90 \%$ and up to $5 \%$ of patients may have unsuspected cirrhosis.(15) As regard FIB-4 index ,73\% of the cases group and $96.7 \%$ of the control group had insignificant fibrosis (F0-F2). $2 \%$ of the 
cases group and no one in the control group had significant fibrosis (F3-F4). $25 \%$ of the cases group and $3.3 \%$ of the control group were indeterminate. There was significant difference between cases group and controls group as shown in table (3). The FIB-4 score, thus, identified only $2 \%$ of patients as having significant fibrosis. This figure is low compared to what is reported in the literature.(16). This means that many cases with significant fibrosis may be missed if we rely upon FIB-4 alone. Worthy of note is the fact that these patients are more in need for active interference to prevent worsening of their condition. Moreover, 25\% of the patients had 'indeterminate' score, which means that they also might lose their chance of being identified and treated.

As regard NAFLD score, $48 \%$ of the cases group and $96.7 \%$ of the control group had insignificant fibrosis (F0-F2). $14 \%$ of the cases group and no one in the control group had significant fibrosis (F3-F4). 38\% of the cases group and $3.3 \%$ of the control group were indeterminate. There was high significant difference between cases group and controls group as shown in table (4). The NAFLD fibrosis score identified $14 \%$ of patients as having significant fibrosis, thus in need for active interference to prevent further progression. This is in agreement
(17) but is slightly different from (18).Consequently , NAFLD fibrosis score is better than FIB-4 score for the monitoring of patients with NAFLD. However, it should be noted that as large as $38 \%$ of cases are 'indeterminate' score, which emphasizes the need for a more efficient method for monitoring in patients with NAFLD.

According to ARFI, 54\% of the cases and $53.3 \%$ of the control showed F0 score. $4 \%$ of the cases and $40 \%$ of the control showed F1 score. $10 \%$ of the cases and $3.3 \%$ of the control showed F2 score. 14\% of the cases and $3.3 \%$ of the control showed F3 score. $18 \%$ of the cases and no one of the control showed F4 score. There was high significant difference between both groups as shown in table (5). Approximately one third of cases with NAFLD are identified by ARFI as having 'significant' fibrosis. This in agreement with (19) but is slightly different from (20). As such, more cases in need for active interference by ARFI than FIB-4 and NAFLD fibrosis score. Moreover, there are no indeterminate results in ARFI. Consequently, ARFI is probably the best method for monitoring patients with NAFLD. It is non-invasive, rapid, repeatable procedure which can be serially monitored to follow management of NAFLD patients at risk of advanced liver disease. It is also 
noticeable that $40 \%$ of healthy controls had F1 fibrosis according to ARFI (Table 5). F1 fibrosis is fibrosis restricted to the portal tracts. This might be due to exposure to schistosomal infection early in life, which is endemic and widely prevalent in Egypt. Mild periportal fibrosis might also be due to exposure to other infections, chemical toxins or food hepatotoxins. (21) .Overall , a wide scale population-based study is needed in Egypt to determine ARFI cut off values specific to the local population. For the time being, ARFI value of $1.185 \mathrm{~m} / \mathrm{s}$ seems too low for F1 in Egypt. On the other hand, it should also be noted that $3.3 \%$ of controls had ARFI F3 fibrosis score. This figure illustrates that clinical, biochemical and conventional imaging methods are not sufficient to prove that the liver is completely healthy. Liver disease can be very subtle in its early stages. As the availability of ARFI expands, it is highly likely that more cases believed to be healthy, will be identified as having subtle liver disease. However, it is also not possible to completely rule out false positive results of ARFI based upon the data available in this study.

The degree of agreement between ARFI and FIB-4 index in table (6) revealed that there is $80 \%$ agreement between both scores. The ROC curve for the performance of ARFI in prediction of F3 or more (taking FIB-4 as a gold standard) shows sensitivity of $100 \%$ and specificity of $79.5 \%$.

The degree of agreement between ARFI and NAFLD fibrosis score in table (7) revealed that there is $87.1 \%$ agreement between both scores. The ROC curve for the performance of ARFI in prediction of F3 or more (taking NAFLD as a gold standard) shows sensitivity of $71.4 \%$ and specificity of $83.3 \%$.

The degree of agreement between FIB-4 and NAFLD fibrosis score in table (8) revealed that there is $63 \%$ agreement between both scores.

It is clear that the concordance agreement between ARFI on one hand and FIB-4 score and NAFLD fibrosis score on the other hand is acceptably high and is statistically significant. Moreover, this concordance agreement is even higher than the agreement between FIB-4 score and NAFLD fibrosis score with each other $(80 \%, 87 \%$ and $63 \%)$. These findings validate the credit which should be attributed to ARFI over the other two 'non-invasive' methods for follow up and monitoring of patients with NAFLD. 
Certainly, liver biopsy is still the universal 'gold standard'. However, the massive number of patients of NAFLD and the known limitations of liver biopsy make it a probitively cumbersome method for follow up and repeated monitoring of 'apparently' healthy individuals.

Table (9) shows relation between grade of fatty liver and ARFI. 30\% of grade I fatty liver, $26.7 \%$ of grade II fatty liver and $37.1 \%$ of grade III fatty liver patients showed significant fibrosis $(>/=F 3)$ according to ARFI. There was no significant relation between them. This finding is very interesting. First, it confirms that the 'brightness' level noticed in ultrasound is actually due to the deposition of fat, not fibrous tissue. Second, it illustrates that the risk of progressive liver disease cannot be gauged by the estimation or monitoring the amount of fat infiltration. This is in agreement with the 'double hit' theory of pathogenesis of NAFLD and NASH. Fat, itself, is not harmful to the liver cells. A second hit factor is needed to trigger progression. Third, it excludes fatty infiltration as a statistical confounding factor to the interpretation of ARFI results in this study. Fourth, it support the conclusion of (22) who stated that the current four point grading system of hepatic steatosis is too simplistic for quantification despite being the most widely used system to evaluate hepatic steatosis in clinical practice.(22)

\section{Conclusion}

The findings demonstrate the superiority of ARFI over the other two 'non-invasive' methods for follow up and monitoring of patients with NAFLD. But unfortunately, liver biopsy is still the universal 'gold standard' in assessing liver fibrosis. However, the large number of patients of NAFLD and the complications of liver biopsy make it inconvenient for follow up and repeated monitoring of healthy individuals.

\section{References:}

1. Masuoka HC, Chalasani N. Nonalcoholic fatty liver disease: an emerging threat to obese and diabetic individuals. Ann N Y Acad Sci. 2013;1281(1):106-22.

2. Ratziu V, Charlotte F, Heurtier A, Gombert S, Giral P, Bruckert E, et al. Sampling variability of liver biopsy in nonalcoholic fatty liver disease. Gastroenterology. 2005;128(7):1898-906.

3. Gennisson J-L, Deffieux T, Fink M, Tanter M. Ultrasound elastography: principles and techniques. Diagn Interv Imaging. 2013;94(5):487-95.

4. Shiina T, Nightingale KR, Palmeri ML, Hall 
TJ, Bamber JC, Barr RG, et al. WFUMB guidelines and recommendations for clinical use of ultrasound elastography: Part 1: basic principles and terminology. Ultrasound Med Biol. 2015;41(5):1126-47.

5. Chalasani N, Younossi Z, Lavine JE, Diehl AM, Brunt EM, Cusi K, et al. The diagnosis and management of non-alcoholic fatty liver disease: Practice guideline by the American Gastroenterological Association, American Association for the Study of Liver Diseases, and American College of Gastroenterology. Gastroenterology [Internet]. 2012;142(7):1592$609 . \quad$ Available from: http://dx.doi.org/10.1053/j.gastro.2012.04.001

6. Angulo P, Hui JM, Marchesini G, Bugianesi E, George J, Farrell GC, et al. The NAFLD fibrosis score: A noninvasive system that identifies liver fibrosis in patients with NAFLD. Hepatology. 2007;45(4):846-54.

7. Sterling RK, Lissen E, Clumeck N, Sola R, Correa MC, Montaner J, et al. Development of a simple noninvasive index to predict significant fibrosis in patients with $\mathrm{HIV} / \mathrm{HCV}$ coinfection. Hepatology. 2006;43(6):1317-25.

8. Yun MH, Seo YS, Kang HS, Lee KG, Kim $\mathrm{JH}, \mathrm{An} \mathrm{H}$, et al. The effect of the respiratory cycle on liver stiffness values as measured by transient elastography. J Viral Hepat. 2011;18(9):631-6.

9. Ferraioli G, Tinelli C, Dal Bello B, Zicchetti M, Filice G, Filice C. Accuracy of real-time shear wave elastography for assessing liver fibrosis in chronic hepatitis C: a pilot study. Hepatology. 2012;56:2125.
10. Fierbinteanu-Braticevici C, Andronescu D, Usvat R, Cretoiu D, Baicus C, Marinoschi G. Acoustic radiation force imaging sonoelastography for noninvasive staging of liver fibrosis. World J Gastroenterol WJG. 2009;15(44):5525.

11. Wieckowska A, Zein NN, Yerian LM, Lopez AR, McCullough AJ, Feldstein AE. In vivo assessment of liver cell apoptosis as a novel biomarker of disease severity in nonalcoholic fatty liver disease. Hepatology. 2006;44(1):27-33.

12. Nightingale $\mathrm{K}$, Bentley R, Trahey G. Observations of tissue response to acoustic radiation force: opportunities for imaging. Ultrason Imaging. 2002;24(3):129-38.

13. Nightingale KR, Palmeri ML, Nightingale RW, Trahey GE. On the feasibility of remote palpation using acoustic radiation force. $\mathrm{J}$ Acoust Soc Am. 2001;110(1):625-34.

14. Collantes RS, Ong JP, Younossi ZM. The metabolic syndrome and nonalcoholic fatty liver disease. Panminerva Med. 2006;48(1):41-8.

15. Boza C, Riquelme A, Ibañez L, Duarte I, Norero E, Viviani $P$, et al. Predictors of nonalcoholic steatohepatitis (NASH) in obese patients undergoing gastric bypass. Obes Surg. 2005;15(8):1148-53.

16. Sumida $\mathrm{Y}$, Yoneda M, Hyogo H, Itoh Y, Ono M, Fujii H, et al. Validation of the FIB4 index in a Japanese nonalcoholic fatty liver disease population. BMC Gastroenterol. 2012;12(1):2 . 
17. Qureshi K, Clements RH, Abrams GA. The utility of the "NAFLD fibrosis score" in morbidly obese subjects with NAFLD. Obes Surg. 2008;18(3):264-70.

18. Treeprasertsuk S, Björnsson E, Enders F, Suwanwalaikorn S, Lindor KD. NAFLD fibrosis score: a prognostic predictor for mortality and liver complications among NAFLD patients. World J Gastroenterol WJG. 2013;19(8):1219.

19. Piscaglia F, Salvatore V, Di Donato R, D'Onofrio M, Gualandi S, Gallotti A, et al. Accuracy of VirtualTouch Acoustic Radiation Force Impulse (ARFI) imaging for the diagnosis of cirrhosis during liver ultrasonography. Ultraschall der Medizin-European J Ultrasound. 2011;32(02):167-75.
20. Bota S, Herkner H, Sporea I, Salzl P, Sirli R, Neghina AM, et al. Meta-analysis: ARFI elastography versus transient elastography for the evaluation of liver fibrosis. Liver Int. 2013;33(8):1138-47.

21. Cave M, Falkner KC, Henry L, Costello B, Gregory B, McClain CJ. Serum cytokeratin 18 and cytokine elevations suggest a high prevalence of occupational liver disease in highly exposed elastomer/polymer workers. J Occup Environ Med Coll Occup Environ Med. 2011;53(10):1128.

22. Webb M, Yeshua H, Zelber-Sagi S, Santo E, Brazowski E, Halpern Z, et al. Diagnostic value of a computerized hepatorenal index for sonographic quantification of liver steatosis. Am J Roentgenol. 2009;192(4):909-14.

To cite this article: Nader S. Anis, Ahmed F. Yousef, Hamada M. Khater, Enas M. El-Kharbotly. Assessment of Liver Fibrosis in Non-Alcoholic Fatty Liver Disease Using Acoustic Radiation Force Impulse. BMFJ 2020; 37 (Radiology): 100-117. DOI:10.21608/bmfj.2020.16442.1044. 